\title{
AIF knockdown induce apoptosis and mitochondrial dysfunction in cochlear spiral ganglion neurons in vitro
}

\author{
LIANG ZONG ${ }^{1,2^{*}}$, JIANDONG ZHAO $^{1,2^{*}}$, WENMING WU $\mathrm{W}^{1,2}$, \\ JIALING WANG $^{1,2}$, DELIANG HUANG ${ }^{1,2}$ and MINGBO LIU ${ }^{1-3}$ \\ ${ }^{1}$ Department of Otolaryngology-Head and Neck Surgery, Institute of Otolaryngology, PLA General Hospital; \\ ${ }^{2}$ China National Clinical Research Center for Otolaryngologic Diseases, Beijing 100853; \\ ${ }^{3}$ Department of Otolaryngology-Head and Neck Surgery, Hainan Branch of PLA General Hospital, \\ Sanya, Hainan 572000, P.R. China
}

Received June 1, 2019; Accepted January 14, 2020

DOI: $10.3892 / \mathrm{mmr} .2020 .10970$

\begin{abstract}
The underlying mechanism involved in auditory neuropathy spectrum disorder (ANSD) remains largely unclear. It has been previously reported that mutations in the apoptosis-inducing factor (AIF) gene are associated with auditory neuropathy and delayed peripheral neuropathy, which can eventually cause ANSD. In the present study, the regulatory effects of AIF knockdown on the cellular functions of spiral ganglion neurons (SNGs) and the molecular mechanism(s) of AIF knockdown in inducing cell apoptosis in SGNs were further investigated. The results showed that the AIF knockdown via siRNA transfection resulted in high levels of oxidative stress, and impaired mitochondrial respiration activity and membrane potential in SGNs. Western blotting further proved that the knockdown of AIF can decrease the content of anti-apoptotic and anti-oxidative proteins, as well as mitochondrial respiratory chain Complex I proteins. The present experimental data suggested that the abnormal expression of AIF may affect SGNs cellular function, and may contribute to the progress of ANSD.
\end{abstract}

\section{Introduction}

Auditory neuropathy spectrum disorder (ANSD) is a specific form of hearing loss that can affect people of all ages $(1,2)$. It is normally characterized by preserved outer hair cell function and abnormal neural conduction of the auditory pathway (1). As a result, the inner ear receives sounds successfully, but the

Correspondence to: Dr Liang Zong, Department of Otolaryngology-Head and Neck Surgery, Institute of Otolaryngology, PLA General Hospital, 28 Fuxing Road, Beijing 100853, P.R. China E-mail: cell-099@163.com

*Contributed equally

Key words: AIF, auditory neuropathy spectrum disorder, siRNA knockdown, cochlear spiral ganglion neurons, cellular dysfunction cochlea has a problem with sending the signals from the ear to the auditory nerve and brain stem (3). Impaired hearing presents as fluctuations in hearing sensitivity, as well as speech perception performance, which can eventually lead to social isolation, depression, and a reduction in professional capabilities, especially for young children.

It has been reported that disorders of the cochlea and auditory neuropathies, such as injured inner hair cells (IHCs), abnormal communication between the IHCs and the auditory nerve, or the impaired function of the cochlear spiral ganglion neurons (SNGs) or auditory nerve itself, may account for the pathophysiological mechanisms of ANSD $(4,5)$. However, the underlying mechanism involved in this pathology remains largely unclear.

Several studies have indicated that apoptosis-inducing factor mitochondrion-associated 1 (AIFM1) gene expressing AIFM1 protein or AIF plays a vital role in triggering chromatin condensation and DNA fragmentation to induce programmed cell death (apoptosis), with a significant role in the mitochondrial respiratory chain and metabolic redox reactions $(6,7)$. As a result, the abnormal expression of AIFM1 gene expression is linked to multiple clinical phenotypes, such as mitochondrial encephalomyopathy, spondyloepimetaphyseal dysplasia with mental retardation, X-linked recessive Charcot-Marie-Tooth disease- 4 and ANSD $(8,9)$. Recently, a high-level association of mutations was reported in the AIF gene in ANSD. Through the use of whole-exome sequencing (WES), it has been shown that variants in the AIFMI gene were the main cause of familial and sporadic ANSD (10). Immunostaining of the murine inner ear also indicated a ubiquitous expression of the AIFM1 gene in IHCs, cochlear outer hair cells (OHCs), and especially SGNs. Among these cells, SGNs are the first afferent neurons in the auditory pathway, which makes them the focus of research. However, the potential regulatory effects of AIF knockdown on the cellular function of SGNs are rarely reported.

The aim of the present study was to further study the potential role of AIF dysfunctions in impaired cochlear SNGs cellular functions. The regulatory effects of AIF on mitochondrial function and cellular apoptosis were revealed. 


\section{Materials and methods}

Tissue culture isolation and detection of AIF expression. The rats were handled in accordance with the NIH Office of Animal Care and Use Animal Research Advisory Committee, and were subjected to the lowest possible levels of pain and discomfort. All animal procedures performed on SPF SD rats were approved by the local Institutional Animal Care and Use Committee of the Chinese PLA General Hospital. Spiral ganglia ( $\mathrm{n}=6$ for each time point) were isolated from SD rats on postnatal days $1(1 \mathrm{~d}), 4(4 \mathrm{~d}), 7(1 \mathrm{w}), 28(4 \mathrm{w}), 56(8 \mathrm{w})$ and postnatal year 2 . The rats were housed in SPF animal center (temperature $20-25^{\circ} \mathrm{C}$ ) under standard acclimatization conditions of $12 \mathrm{~h}$ light/dark cycle and with ad libitum access to water and a commercial standard chow. Health and behavior monitoring by visual examination was performed daily.

The isolation procedures were performed as previously described (11-13). Briefly, for neonatal SD rats (up to 10 days of age), the animals were anesthetized via an intravenous injection of $30 \mathrm{mg} / \mathrm{kg}$ sodium pentobarbital and then decapitated. For SD rats older than 10 days, the rats were placed in the euthanasia chamber with readily visible and $100 \%$ carbon dioxide was introduced, during which the flow rate must displace no more than $30 \%$ of the chamber volume/minute. The expected time to unconsciousness is usually within 2 to $3 \mathrm{~min}$. After observation of each rat for signs of both lack of respiration and faded eye color, the rat was removed from the cage for further tissue isolation.

During the tissue isolation, the skulls were opened along the midsagittal (median) plane with a surgical scissor. Temporal bones from both sides were cut off and placed into in ice-cold sterile PBS with 2\% BSA and 2\% glucose under a stereomicroscope. The cochlear capsule was separated using fine forceps to expose the membranous labyrinth. To avoid cross-contamination, the region where the ganglia were in contact one another was discarded.

To detect the mRNA expression levels of AIF, RNA isolated from the cochlear capsule tissue was measured by RT-qPCR. Briefly, total RNA of the micro-dissected samples was isolated by using an Omega Total RNA kit I (Omega Bio-Tek, Inc., Norcross, GA, USA), according to the manufacturer's instructions. cDNA was synthesized using Takara PrimeScript RT reagent kit with gDNA Eraser (Takara Biotechnology Co., Ltd., Dalian, China), according to the manufacturer's instructions. RT-qPCR analysis of the RNA was performed using a SYBR Premix EX Taq kit (Takara Biotechnology Co., Ltd.) and RNase-free 96-well PCR plates under the following cycling conditions: 40 Cycles of $95^{\circ} \mathrm{C}$ for $15 \mathrm{sec}$ and $58^{\circ} \mathrm{C}$ for $30 \mathrm{sec}$.

The forward and reverse primers (Sangon Biotech Co., Ltd., Shanghai, China) were used for the specific RNAs in RT-qPCR analysis: AIF, Forward: 5'-CGGCGGTGTGTG AAAAGAAA-3' and 5'-GGAGTTCTAGAGGAACACGCC-3' reverse (199 bp); $\beta$-actin, Forward 5'-AACCTTCTTGCAGCT CCTCC-3' and 5'-CCTTCTGACCCATACCCACC-3' reverse (202 bp). $\beta$-Actin gene expression was used for normalization and three independent experiments were performed for each time points.

Western blotting. The cochlear capsule tissue $(\mathrm{n}=3)$ was lysed with Laemmli Sample Buffer (Bio-Rad Laboratories,
Inc., Hercules, CA, USA). BCA Protein Assay kit (Beyotime Institute of Biotechnology, Shanghai, China) was used to measure the total protein content of the lysates. The lysates samples were further heated at $100^{\circ} \mathrm{C}$ for $10 \mathrm{~min}$ and loaded onto $10 \%$ sodium dodecyl sulfate polyacrylamide gels (SDS-PAGE) with equal amounts of total protein. After being electro-transferred to polyvinylidene fluoride (PVDF) membranes (Bio-Rad Laboratories, Inc.), the samples were blocked with $1 \mathrm{X}$ TBST with $5 \%$ w/v nonfat dry milk at room temperature for $1 \mathrm{~h}$. The membranes were incubated with primary antibodies overnight at $4^{\circ} \mathrm{C}$. Rabbit monoclonal anti-AIF (1:1,000; Abcam, Cambridge, MA, USA), and anti- $\beta$-actin (1:4,000; Beyotime Institute of Biotechnology) primary antibodies were used. The blots were then exposed to the horseradish peroxidase-labelled secondary anti-rabbit antibody $(1: 8,000)$ for $2 \mathrm{~h}$ at room temperature. The proteins were visualized using an enhanced chemiluminescence kit (Thermo Fisher Scientific, Inc., Waltham, MA, USA). The band signals were detected using a gel imaging system (Syngene, Frederick, MD, USA). The intensities were quantified using ImageJ software and the relatively expression levels of certain proteins were calculated via band intensity normalization.

SGNs isolation and culture. SGNs were isolated from the micro-dissected cochlear capsule tissue of newborn rats (1 day) as described above. The tissues in tubes were dissociated in $0.05 \%$ trypsin, $0.37 \mathrm{mM}$ EDTA, $5 \mu \mathrm{g} / \mathrm{ml}$ each of collagenase type 4 each, papain, and dispase, and 2,800 $\mathrm{U} / \mathrm{ml}$ DNase in $1 \mathrm{ml}$ PBS. The tubes were rotated at $37^{\circ} \mathrm{C}$ for $45 \mathrm{~min}$, with gentle trituration using a pipette every $15 \mathrm{~min}$. After $45 \mathrm{~min}$ of enzyme digestion, the tissue was finally triturated 5-10 times and centrifuged for $10 \mathrm{~min}$ at $4^{\circ} \mathrm{C}$ at $800 \mathrm{x} \mathrm{g}$. After discarding the dissociation medium, the cells were re-suspended gently in Dulbecco's modified Eagle's medium supplemented with B272 $\mathrm{ml} / \mathrm{ml}$, BDNF $10 \mu \mathrm{g} / \mathrm{ml}$ and penicillin 100,000 U/1 $1 \%$ (all from Merck KGaA, Darmstadt, Germany) and cultured at $37^{\circ} \mathrm{C}$ in a humidified incubator with $5 \% \mathrm{CO}_{2}$. Cell adhesion and spreading were observed after 12 and $48 \mathrm{~h}$ of culture $(14,15)$.

Immunofluorescence. NF200 immunofluorescence was used to identify the isolated SGNs (16). Following culture for $48 \mathrm{~h}$, the SGNs were fixed in $4 \%$ paraformaldehyde for $20 \mathrm{~min}$ and exposed to $0.2 \%$ Triton X-100 in PBS for permeabilization. The cells were then washed with PBS and incubated with 5\% BSA blocking solution for 20 min. Following incubation with a rabbit anti-NF200 antibody (1:200; Abcam) overnight at $4^{\circ} \mathrm{C}$, the cells were further treated with Alexa-488-conjugated goat anti-rabbit (1:200; Sangon Biotech Co., Ltd.) to label the primary antibodies for $30 \mathrm{~min}$ at $37^{\circ} \mathrm{C}$. The staining results were observed with a fluorescence microscope (Olympus Corporation, Tokyo, Japan).

siRNA knockdown of AIF in SGNs. To verify the role of AIF in SGNs, we firstly performed siRNA knockdown of AIF using siRNA oligonucleotides (oligos) targeting the coding region of AIF. Following culture for $24 \mathrm{~h}$, the SGNs were transfected with siRNA via Amaxa 4D nucleofector (Lonza Group, Ltd. Basel, Switzerland) using P4 Primary Cell 4D-Nucleofector ${ }^{\circledR}$ $\mathrm{X}$ kit L (Lonza Group, Ltd.), according to the manufacturer's instructions. Half of the medium was then replaced with fresh 
culture medium after $4 \mathrm{~h}$ and the cells were incubated in a humidified incubator with $37^{\circ} \mathrm{C} / 5 \% \mathrm{CO}_{2}$ until analysis. The siRNA-AIF oligos for AIF targeting were as follows: Sense 5'-CAGAAGGAGGCUCAGUUCCUCCAAUdTdT-3', and anti-sense 5'-AUUGGAGGAACUGAGCCUCCUUCUGdT dT-3'; and siRNA-control oligos for control experiments, Sense 5'-GCAUCGAAUAUUCUACCUUdTdT-3', and Anti-Sense 5'-AAGGUAGAAUAUUCGAUGCdTdT-3' were used. siRNA (15 pmol/sample) was used, and normal siRNA was replaced of with Cy3 labeled siRNA for observation of the transfection efficiency. The AIF protein contents were analyzed by western blotting. The SGNs cultured on tissue culture polystyrene (TCPS) without further treatment were termed the TCPS group, which was used as the blank comparison group.

Intracellular reactive oxygen species (ROS) level detection. To evaluate the regulatory effect of the AIF siRNA knockdown on cellular oxidative stress status, the intracellular ROS was monitored using 2',7'-dichlorofluorescein diacetate (DCFH-DA). Forty-eight hours after siRNA transfection, the SGNs were treated with DCFH-DA $(10 \mu \mathrm{M})$ at $37^{\circ} \mathrm{C}$ for 20 min in the dark. Next, flow cytometry (BD FACS Calibur; Becton, Dickinson and Company, Franklin Lakes, NJ, USA) was performed to measure the fluorescence intensity of the treated cells. The fluorescence of the oxidation product of DCFH-DA, DCF, was excited at $488 \mathrm{~nm}$ and detected at $535 \mathrm{~nm}$ by flow cytometry. Data was acquired and analyzed using the CELL Quest programme PRO software (Becton, Dickinson and Company). The percentage of cells with a high DCF fluorescence of $10^{3}-10^{5}$ units, as measured by flow cytometry, was used to indicate the intracellular (ROS) level.

The staining fluorescence of SGNs was also observed with a fluorescent microscope (Leica Microsystems $\mathrm{GmbH}$, Wetzlar). Images from 4 random fields in each group were recorded and the fluorescence density was quantified with ImagePro Plus ${ }^{\mathrm{TM}}$ software.

The intracellular malondialdehyde (MDA) levels were determined by using a Lipid Peroxidation MDA Assay kit (Beyotime Institute of Biotechnology). To determine the intracellular malondialdehyde (MDA) levels, the SGNs were lysed with cell lysis buffer and the total protein content of the lysates was measured using BCA Protein Assay kit (Beyotime Institute of Biotechnology). The MDA levels of cell lysis with equal protein contents were detected according to the manufacturer's instructions and expressed as $\mu \mathrm{mol} / \mathrm{mg}$ protein.

Mitochondrial complexes activity and adenosine triphosphate (ATP) generation measurement. Twenty-four hours after transfection, cells were collected and suspended in PBS (pH 7.2). They were then split through 3 of freeze/thawing cycles. The activity of complex I, II/III and IV were determined spectrophotometrically using Mitochondrial complex I, II/III and IV Assay kits, respectively (Genmed Scientifics Inc., Arlington, MA, USA), according to the manufacturer's instructions.

In order to determine ATP concentrations, a colorimetric ATP assay kit (ab83355, Abcam, England) was used. The cells were lysed according to the manufacturer's instructions and were placed into a 96-well plate. The absorbance of the reaction mixture was measured at $550 \mathrm{~nm}$ (17).
Mitochondrial membrane potential. The mitochondrial membrane potential $(\Delta \psi \mathrm{m})$ was assessed using MitoProbe ${ }^{\mathrm{TM}}$ DiIC1 (5) according to the MitoProbe ${ }^{\mathrm{TM}}$ DiIC1 (5) Mitochondrial Membrane Potential Protocol. The cells were analyzed by flow cytometry with a $633 \mathrm{~nm}$ excitation using emission filters appropriate for Alexa Fluor 633 dye. The percentages of cells with a high DiIC1 fluorescence ( $>10^{2}$ units) measured by flow cytometry indicated the mitochondrial membrane potential level.

Determining of protein contents. The proteins contents of SGNs $24 \mathrm{~h}$ after transfection were determined by western blotting. Immunoblotting was performed using the following primary antibodies: Anti-cytochrome complex (Cyt C) $(1: 2,000)$, anti-Bcl-2 (1:1,000), anti-Bax (1:1,000), anti-poly(ADP-ribose) polymerase (PARP)-1, anti-superoxide dismutase 1 (SOD)-1 (1:1,000), anti-SOD-2, anti-Caspase-3 (Casp-3) (1:1,000), anti-C I 39 kDa (1:1,000), anti-C I 20 kDa (1:1,000), anti-C I $17 \mathrm{kDa}(1: 1,000)$, anti-C III $49 \mathrm{kDa}(1: 1,000)$, anti-C III $47 \mathrm{kDa}$ (1:1,000), anti-C IV $20 \mathrm{kDa}(1: 1,000)$, anti-PAR (1:500), anti-GAPDH $(1: 8,000)$, anti- $\beta$-actin $(1: 10,000)$, anti-tubulin $(1: 10,000)$, anti-Histone H3 $(1: 1,000)$ and anti-COX IV $(1: 1,000)$, which were bought from Cell Signaling Technology, Inc., Danvers, MA, USA.

For nuclear, mitochondrial and cytosolic protein enrichment, the cells were fractionated into nuclear, mitochondrial and cytosolic fractions using the Focus SubCell kit (Geno Technology, Inc., St. Louis, MO, USA). Briefly, the treated cells were first harvested and washed in ice-cold SubCell Wash Buffer and then re-suspended in ice cold FOCUS ${ }^{\mathrm{TM}}$ SubCell Buffer-I. Following mechanic lysis and the addition of SubCell Buffer-II, the lysed cells were centrifuged for $10 \mathrm{~min}$ at $700 \mathrm{x} g$ to pellet the nuclei. The nuclear pellet was further enriched by gradient centrifugation and used for further experiments. The cytosolic supernatant with mitochondria was further centrifuged at $12,000 \mathrm{x} \mathrm{g}$ for $15 \mathrm{~min}$ at $4^{\circ} \mathrm{C}$ to pellet the mitochondria for protein content detection. Finally, the cytosolic supernatant was removed and placed into a fresh tube. The enriched proteins were analyzed by SDS-PAGE and western blotting. The relative protein expression levels were calculated by band intensity normalization to internal control, and COX IV, Tubulin and histone $\mathrm{H} 3$ are used as internal control for the mitochondrial, cytoplasmic and nuclear fractions respectively (18-20).

Statistical analysis. Quantitative data are expressed as the means \pm standard deviations (mean $\pm \mathrm{SD}$ ). Statistical analysis was performed using one-way ANOVA followed by Tukey's post-hoc test for multiple comparisons (using SPSS software). A P<0.05 was considered to indicate a statistically significant difference.

\section{Results}

AIF expression decreased during the stage of growth and development. To characterize the potential role of AIF in the auditory pathway, we firstly determined the expression levels of AIF in cochlea spiral tissue from rats at different growth phases. As shown in Fig. 1A and B, RT-qPCR results indicated that the mRNA expression levels of AIF decreased over time during the stage of growth and development. However, the AIF 

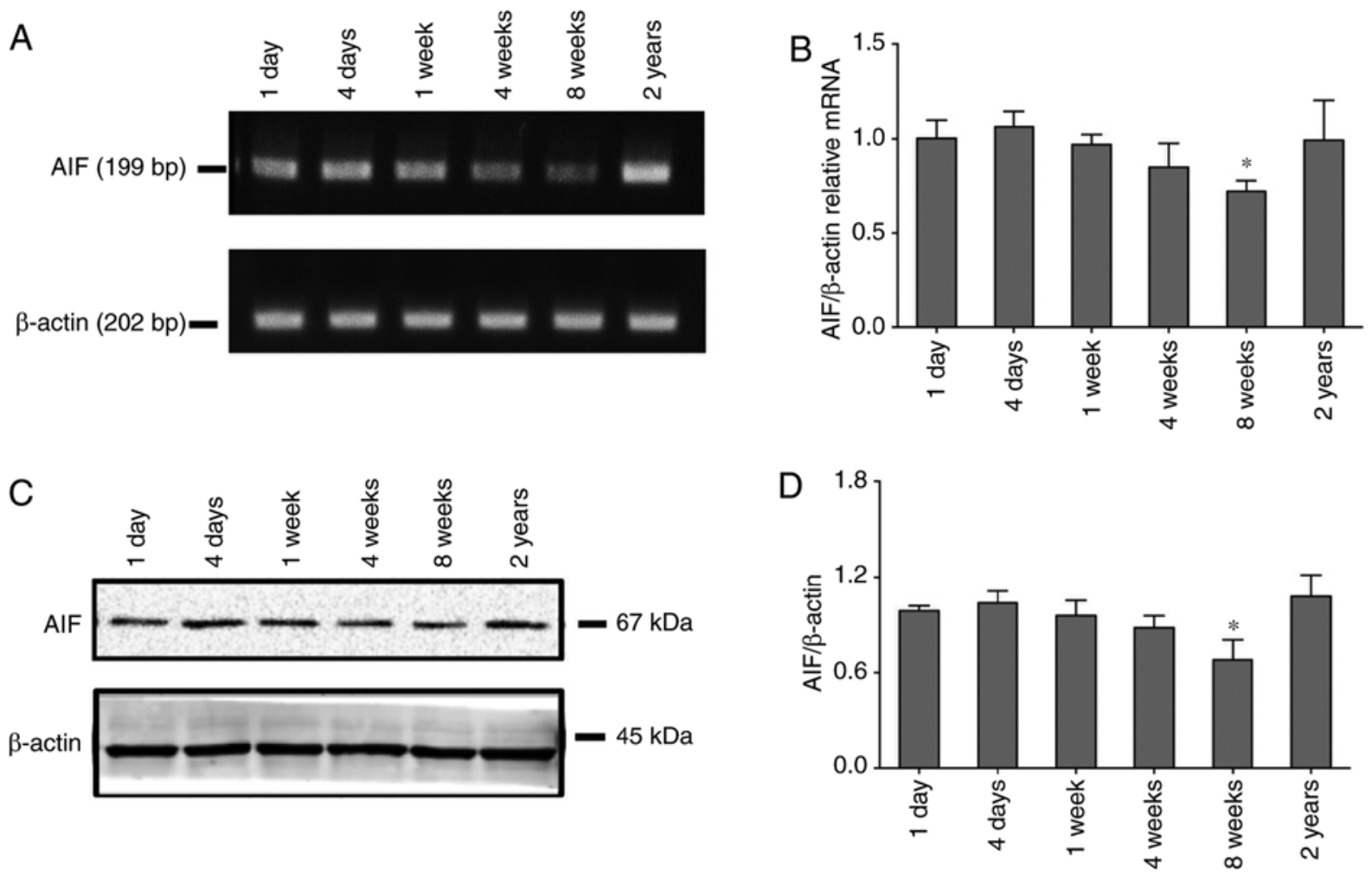

Figure 1. Measurement of AIF expression. (A) RT-qPCR result as detected by gel electrophoresis and (B) RT-qPCR detection of AIF mRNA expression levels in cochlear capsule tissue ( $\mathrm{n}=6$ ). (C) Western blotting of AIF content in cochlear capsule tissue and (D) its quantitative analysis ( $\mathrm{n}=6$ ). ${ }^{*} \mathrm{P}<0.05$ vs. 1 day. AIF, apoptosis-inducing factor.

expression levels in elderly rats were increased to a certain extent. Western blotting (Fig. 1C and D) results of AIF protein contents confirmed a similar trend of the change.

Isolation and identification of SGNs. Fig. 2A illustrates an example of cultured SGNs with no obvious neurite after $12 \mathrm{~h}$ of culture in primary growth medium. More neurites emanated from the cell body after $48 \mathrm{~h}$ of culture. Immunofluorescence results indicated that the surviving SGNs were mostly stained with primary anti-NF200 antibody (Fig. 2B) (21).

AIF knockdown increases the oxidative stress status of $S G N s$. To verify the role of AIF in SGNS apoptosis execution, AIF siRNA knockdown was performed using siRNA oligos targeting the coding region of AIF. As shown in Fig. 3A, the siRNAs labeled with Cy3 fluorescent dye were transfected into SGNs at $24 \mathrm{~h}$ after treatment. Compared to normal SGNs or a control siRNA oligo, AIF knockdown significantly decreased the AIF contents in SGNs (Fig. 3B and C).

Twenty-four hours after siRNA knockdown, the levels of reactive oxygen species (ROS) were measured by the cell-permeant fluorescent indicator 2',7'-dichlorodihydroflu orescein diacetate (DCFH-DA). Following treatment with DCFH-DA, intracellular ROS levels were evaluated by flow cytometry and a fluorescent microscope (Fig. 4A-C). As compared to normal SGNs in the TCPS group and control siRNA oligo in the siRNA-control group, AIF knockdown in SGNs significantly increased their intracellular ROS levels.

MDA is a mutagenic product of lipid peroxidation, which is closely associated with intracellular oxidative stress levels.
A
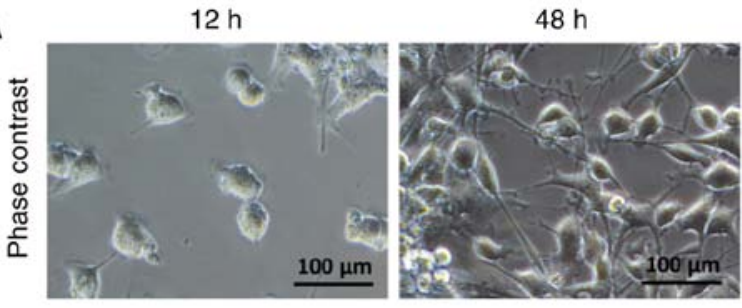

B
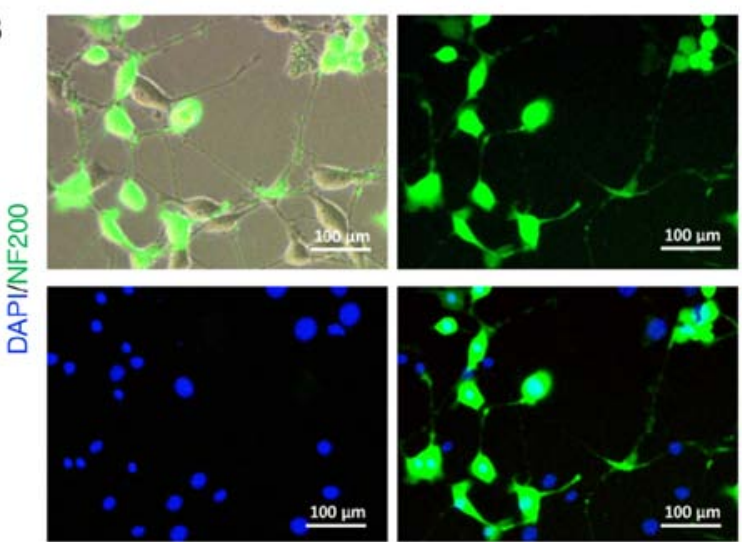

Figure 2. SGN culture and identification. (A) Representative image of SGNs after a 12- and 48-h culture. (B) Identification of SGNs by anti-NF200 immunofluorescent staining. SNG, spiral ganglion neuron.

Generally, cellular MDA levels are low in SGNs, but AIF knockdown could significantly increase them (Fig. 4D), which also confirmed the high oxidative stress status in the siRNA-AIF group. 
A

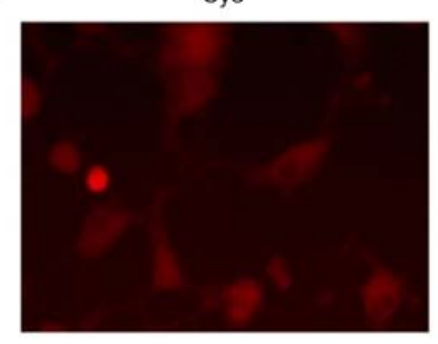

в

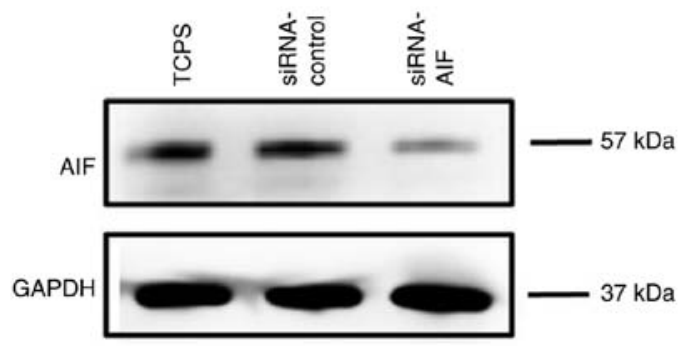

Phase-contrast

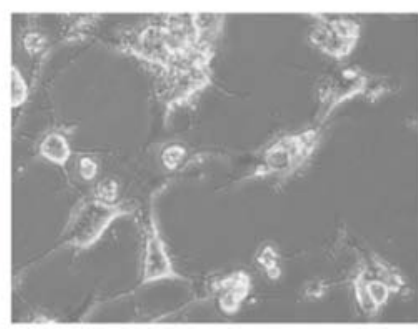

C

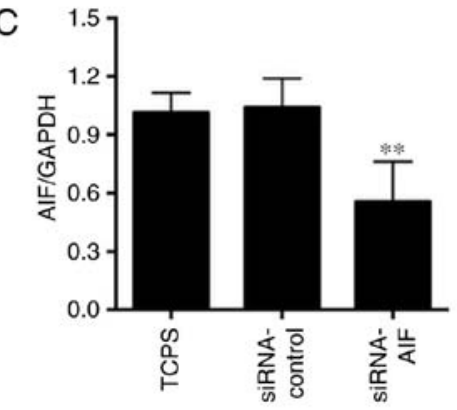

Figure 3. Transfection of SGNs with siRNA to knockdown AIF expression. (A) Observation of the Cy3-labeled siRNAs $24 \mathrm{~h}$ after their transfection into SGNs. (B) Western blotting of AIF content in SGNs $24 \mathrm{~h}$ after transfection and (C) its quantitative analysis $(\mathrm{n}=6) .{ }^{* *} \mathrm{P}<0.01 \mathrm{vs}$. TCPS and siRNA-control. SNG, spiral ganglion neuron; AIF, apoptosis-inducing factor.

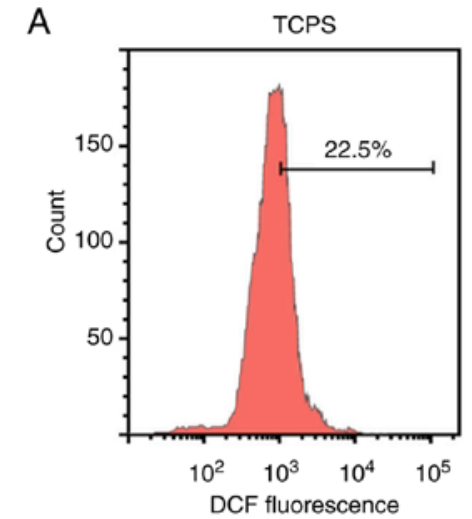

B
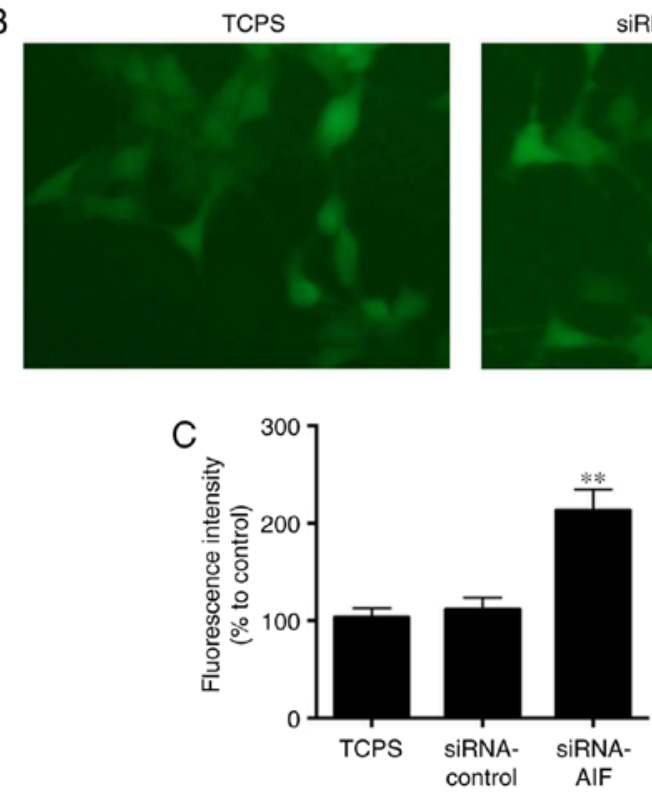
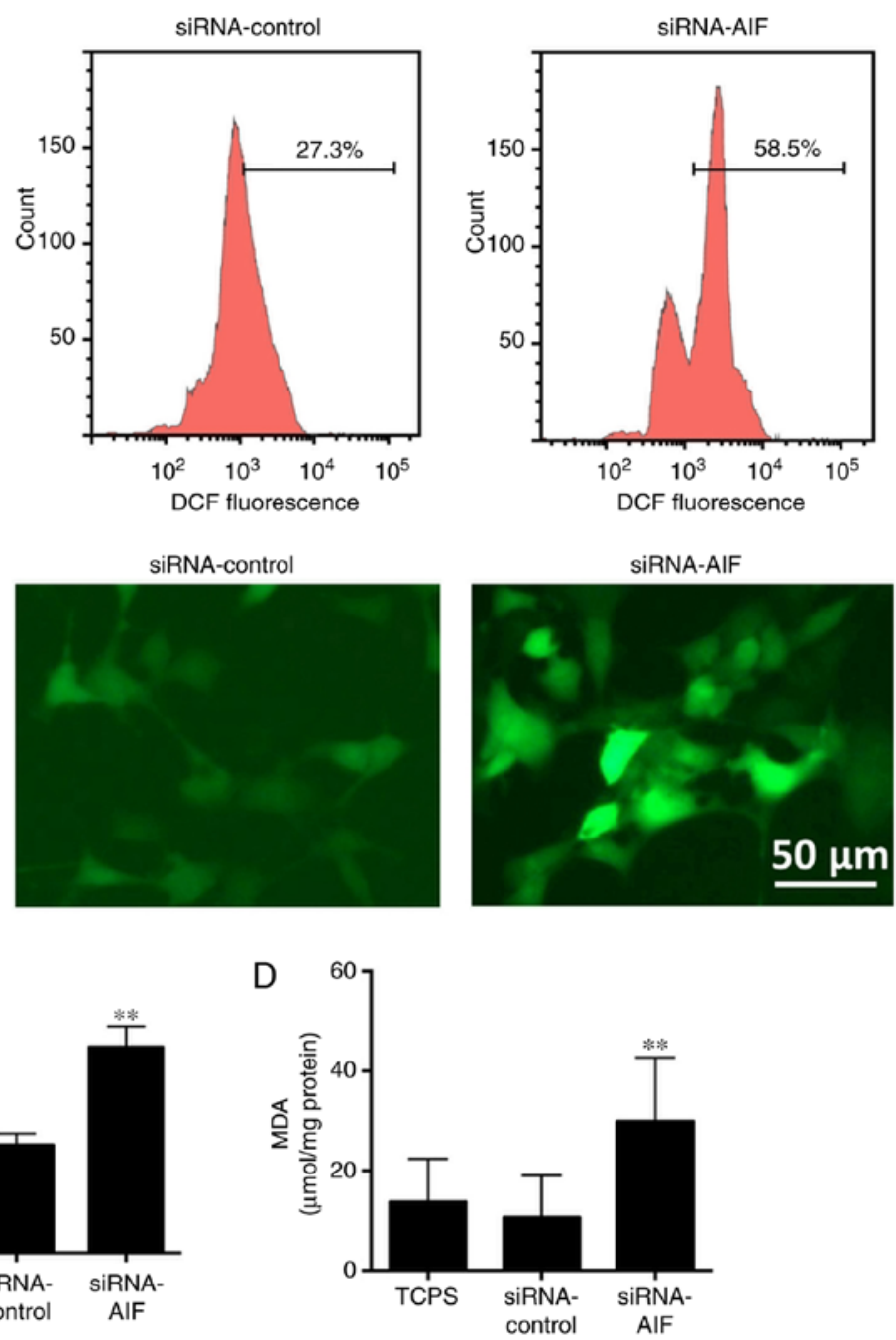

Figure 4. AIF knockdown increases oxidative stress in SGNs. (A) Flow cytometry, (B) fluorescence microscope observation of DCFH-DA staining and (C) quantitative analysis of its fluorescence intensity ( $\mathrm{n}=12)$. (D) MDA detection of lipid peroxidation in $\mathrm{SGNs}(\mathrm{n}=12) .{ }^{* *} \mathrm{P}<0.01 \mathrm{vs}$. TCPS and siRNA-control. SNG, spiral ganglion neuron; DCFH-DA, 2',7'-dichlorofluorescein diacetate. 

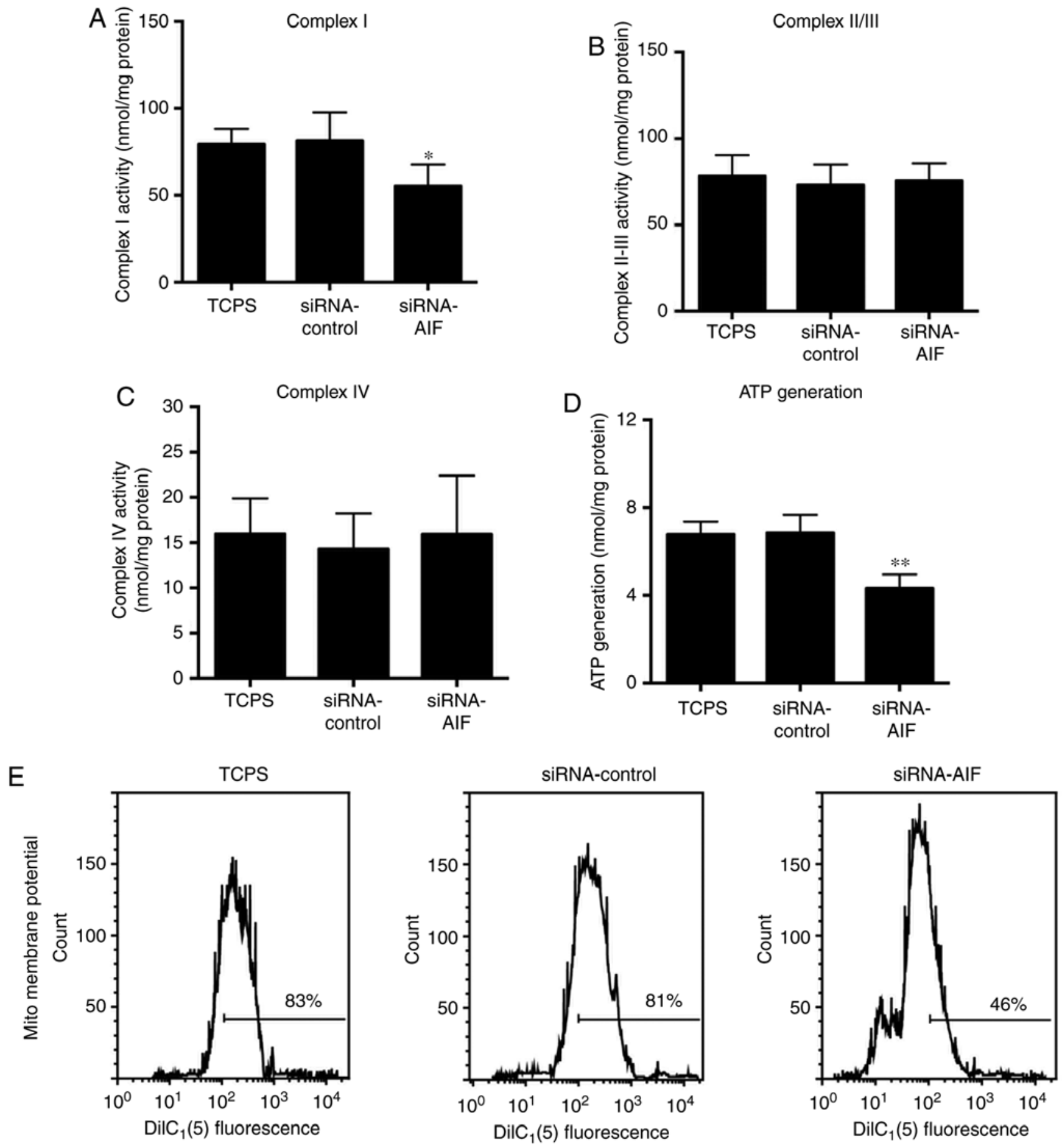

Figure 5. AIF knockdown attenuated mitochondrial respiratory functions and ATP generation. Specific activities of mitochondrial (A) complex I (rotenone-sensitive NADH-ubiquinone oxidoreductase) (n=12), (B) complex II/III (succinate-cytochrome c oxidoreductase) (n=12), (C) complex IV (cytochrome c oxidase) $(\mathrm{n}=12)$ and (D) ATP generation $(\mathrm{n}=12)$. (E) Flow cytometry results by MitoProbe ${ }^{\mathrm{TM}}$ DiIC1 detection of mitochondrial membrane potential. ${ }^{*} \mathrm{P}<0.05$ and ${ }^{* *} \mathrm{P}<0.01$ vs. TCPS and siRNA-control. ATP, adenosine triphosphate.

AIF knockdownattenuated mitochondrial respiration and ATP production. Mitochondrial dysfunction is widely implicated in cells dysfunction through impaired ATP synthesis. Since mitochondrial complex I is a major source of mitochondrial ROS (22), the specific activity of complex I in SGNs was also analyzed. As shown in Fig. 5A and D, complex I activity and ATP was significantly decreased following AIF knockdown, yet similar levels of mitochondrial complex II/III (Fig. 5B) and mitochondrial complex IV (Fig. 5C) were observed between the three groups. Flow cytometry results (Fig. 5E) of mitochondrial membrane potential revealed that AIF knockdown markedly decreased the mitochondrial membrane potential of SGNs.

AIF knockdown impairs SGNs functions through anti-oxidative and anti-apoptotic bioactivities. To verify whether AIF knockdown had an effect on anti-oxidative and apoptotic-related factors, western blotting was performed. As 

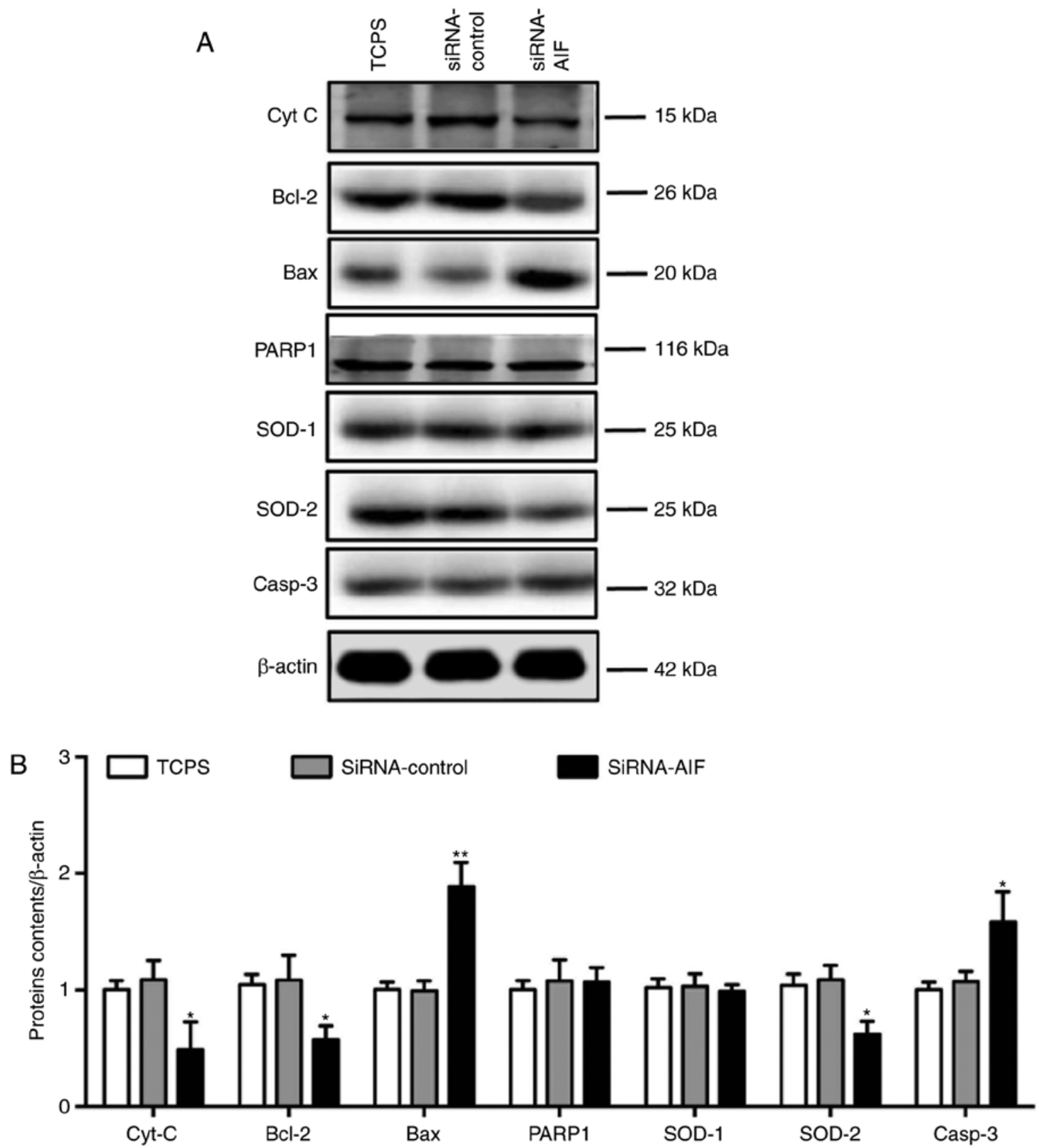

Figure 6. Western blotting of anti-oxidative and anti-apoptotic proteins. (A) Western blotting and (B) quantitative analysis indicated that AIF knockdown in SGNs decreased the expression of anti-apoptotic factors Cyt $\mathrm{C}$ and Bcl-2, and increased that of pro-apoptotic proteins Bax and Casp-3 (n=6). "P<0.05 and ${ }^{* *} \mathrm{P}<0.01$ vs. TCPS and siRNA-control. Cyt C, cytochrome complex; AIF, apoptosis-inducing factor; SGN, spiral ganglion neuron.

shown in Fig. 6A and B, AIF knockdown in SGNs resulted in the decrease of anti-apoptotic factors cytochrome complex (Cyt C) and Bcl-2, and the increase of pro-apoptotic proteins Bax and Caspase-3 (Casp-3). Although similar contens of PARP and SOD-1 were observed between the three groups, anti-oxidative factor SOD-2 in SGNs was decreased after in SGNs following AIF knockdown. Western blotting analysis (Fig. 7A and B) of mitochondrial respiratory complex-related proteins indicated that AIF knockdown decreased the complex I proteins Complex I $39 \mathrm{kDa}$ and Complex I $20 \mathrm{kDa}$, but not Complex I $17 \mathrm{kDa}$, Complex III $49 \mathrm{kDa}$, Complex III $47 \mathrm{kDa}$ and Complex IV $20 \mathrm{kDa}$.

Of note, although AIF knockdown led to a decrease in the AIF protein content in SGNs, western blotting (Fig. 8A and B) of enriched mitochondrial, nuclear and cytosolic proteins indicated that mitochondrial siRNA-AIF was decreased, while nuclear and cytosolic siRNA-AIF were observed with the increase in AIF protein. Similar levels of PARP-1 expression was observed in the nuclei of the three groups (Fig. 8A and C). However, the cytosolic and nuclear poly(ADP-ribose) (PAR) polymer levels were increased following AIF knockdown (Fig. 8A and D).

\section{Discussion}

An improved understanding of the underlying mechanisms of ANSD is essential for the development of new prevention and treatment mechanisms. Previously studies have suggested that 
A
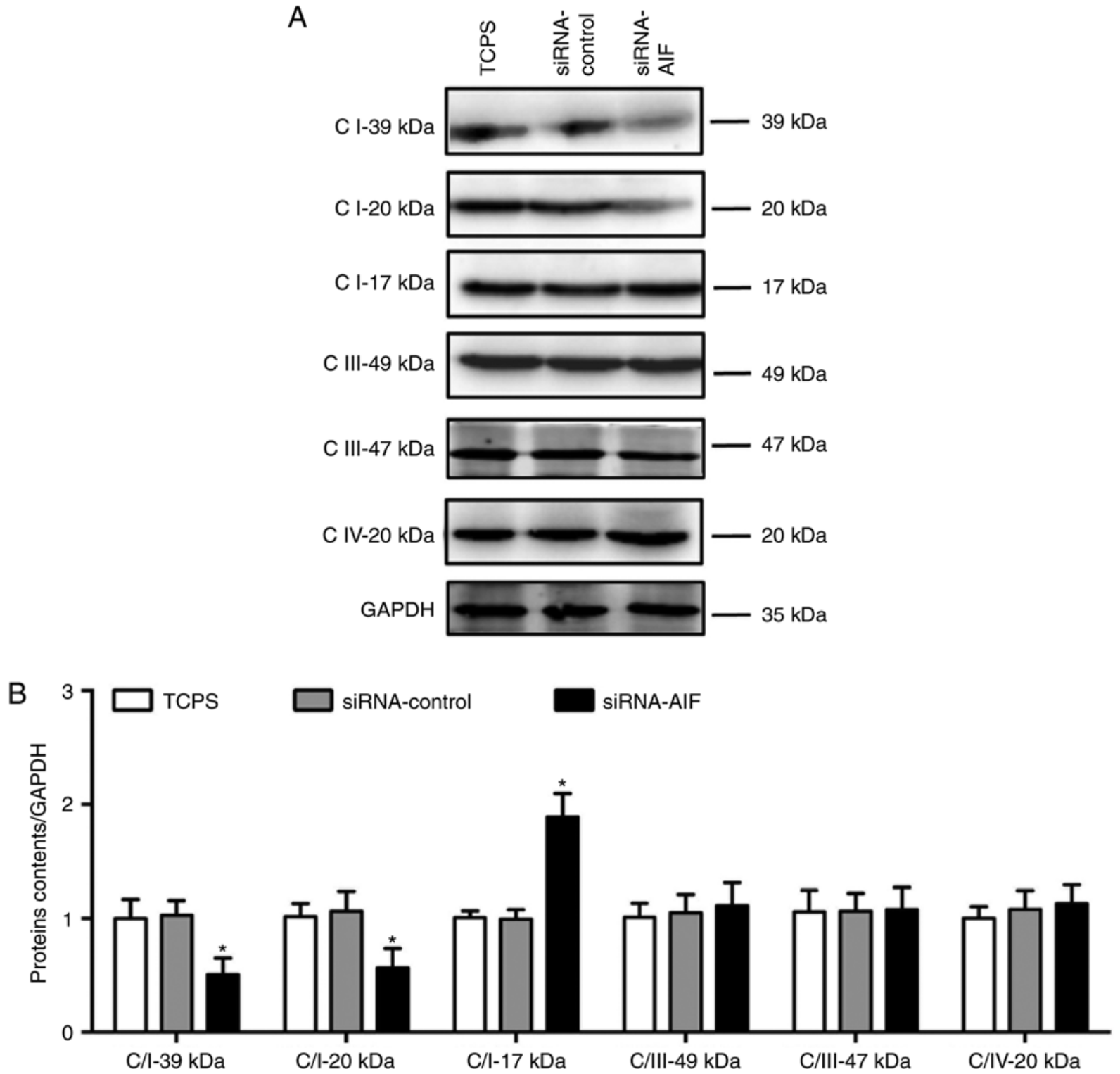

Figure 7. Western blotting of mitochondrial respiratory complex-related proteins. (A) Western blotting and (B) quantitative analysis indicated that AIF knockdown in SGNs decreased the complex I proteins complex I $39 \mathrm{kDa}$ and complex I $20 \mathrm{kDa}$, but not complex I $17 \mathrm{kDa}$, complex III $49 \mathrm{kDa}$, complex III $47 \mathrm{kDa}$ and complex IV $20 \mathrm{kDa}(\mathrm{n}=6)$. " $\mathrm{P}<0.05$ vs. TCPS and siRNA-control. AIF, apoptosis-inducing factor; SGN, spiral ganglion neuron.

the impaired cellular functions of SGNs in the cochlea play vital roles in the progression of hearing loss, but the potential mechanism involved remains largely unclear. The pathological factors inducing programmed cell death (apoptosis) may be the potential mechanism underlying SGNs dysfunction and cellular injury. The results the present study revealed new regulatory effects of the AIF protein on SGNs dysfunctions in vitro. The knockdown of the AIF expressions with siRNA resulted in high levels of oxidative stress status, impaired mitochondrial respiration activity and membrane potential in SGNs. Western blotting analysis further indicated that AIF knockdown could decrease the expression levels of anti-apoptotic and anti-oxidative executors, as well as mitochondrial respiratory chain Complex I proteins.

AIF is an NAD(P)H oxidase with oxidoreductase activity. AIF was first identified as a flavoprotein and acts as a mitochondrial effector of apoptosis. Studies have indicated that AIF plays various roles in apoptosis, electron transport, and ferredoxin metabolism, etc. Furthermore, the silencing of the AIF gene was found to increase ROS generation in the mitochondrion (15). ROS overproduction induced high level oxidative stress status, which plays pivotal roles in various pathologies, especially the apoptosis of impaired cells. In the present study, we firstly determined the expression levels of AIF in cochlea spiral tissue from rats at the different growth phase to primary characterize the potential role of AIF in auditory pathway. The results indicated the decreased AIF expression levels of rats as it grows older (during the growth and development stage). Thus, we further investigated the potential role of AIF knockdown in cochlear spiral ganglion neurons (SNGs) cellular functions. As a result, AIF knockdown resulted in a significant overproduction of ROS, as proven by DCFH-DA staining, and high levels of lipid peroxidation, as measured by MDA. Western blotting results showed that anti-apoptotic executors $\mathrm{Cyt} \mathrm{C}$ and $\mathrm{Bcl}-2$, and anti-oxidative executor SOD-2, were decreased in SGNs by AIF knockdown, 
A
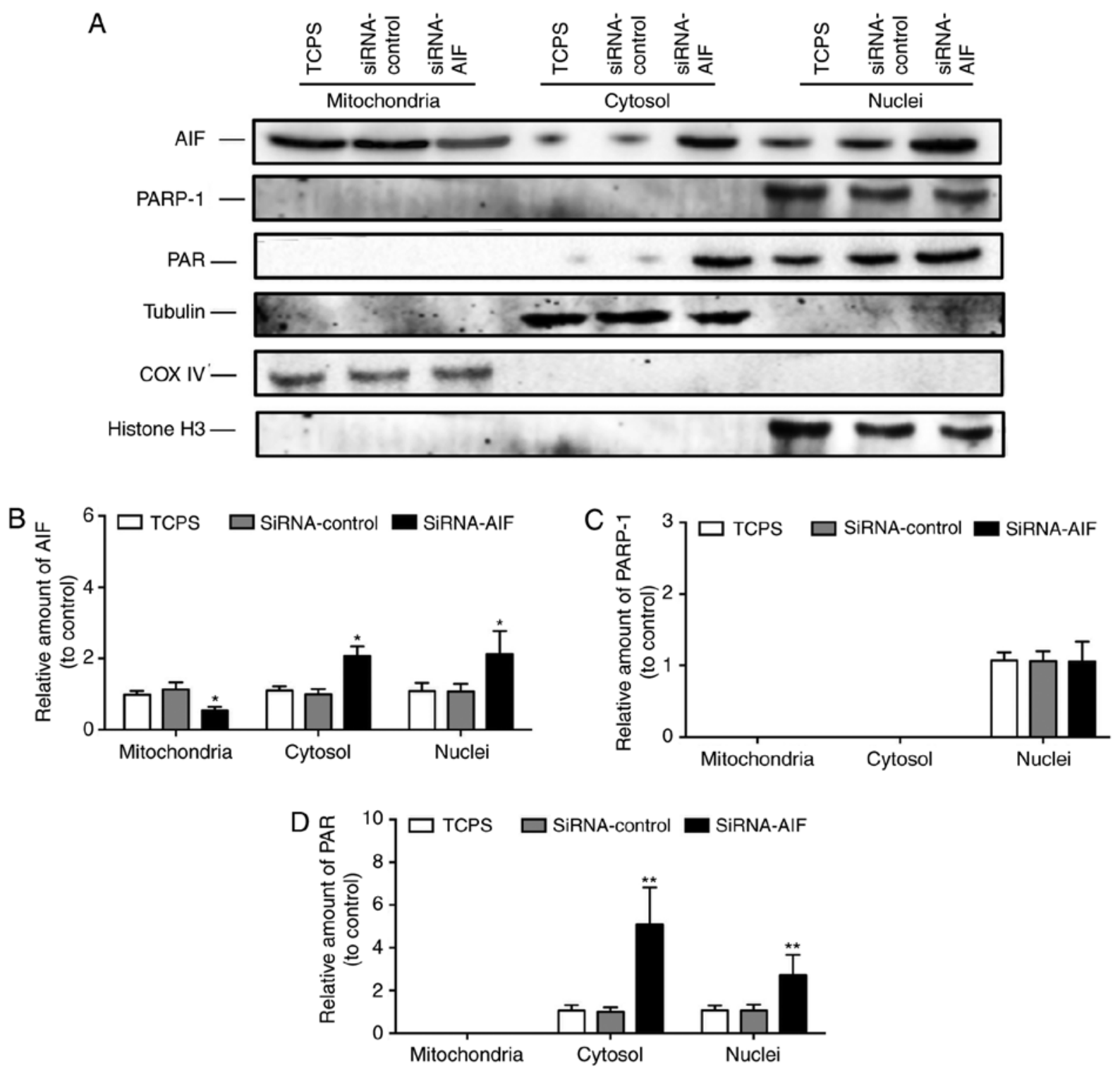

Figure 8. Western blotting of AIF, PARP-1 and PAR. (A) Western blotting and (B-D) quantitative analysis in enriched mitochondrial, nuclear and cytosolic proteins. (A and B) The results proved that the knockdown of AIF resulted in decreased mitochondrial AIF, but increased nuclear and cytosolic AIF. (A and C) Similar levels of PARP-1 expression was observed in the nuclei of the three groups. (A and D) The cytosolic and nuclear PAR polymer levels were increased following AIF knockdown ( $\mathrm{n}=6)$. ${ }^{*} \mathrm{P}<0.05$ and ${ }^{* *} \mathrm{P}<0.01$ vs. TCPS and siRNA-control. The relative protein expression levels were calculated by band intensity normalization to internal control; COX IV for mitochondrial fractions, Tubulin for cytoplasmic fractions and histone $\mathrm{H} 3$ for nuclear fractions respectively. AIF, apoptosis-inducing factor; PAR, poly(ADP-ribose); PARP-1, poly(ADP-ribose) polymerase.

while pro- apoptotic protein Bax and Casp-3 were increased. The high oxidative stress status could potentially lead to injury and apoptosis in SGNs, which may result in dysfunctions of SGNs.

Normally, the flavoprotein AIF is contained in the mitochondrial intermembrane space or inner mitochondrial membrane (23). Upon the induction of apoptosis, AIF translocates from the mitochondria to the cytosol, and rapidly relocalizes to the nuclear compartment, where it can mediate the fragmentation of large-scale DNA (24). Although AIF is not a component of complex I, AIF-deficiency has been proven to reduce complex I content, indicating its important roles in the biogenesis and/or maintenance of mitochondrial respiratory chain complexes function (25). The present results indicated that AIF knockdown decreased the complex I activity, ATP generation and mitochondrial membrane potential in SGNs.
These results suggested that AIF knockdown may induce the mitochondrial dysfunction.

Of note, although it remains unknown what it is that can regulate the release of AIF, in the present study, the data suggested that, even though AIF knockdown resulted in a decrease of the total AIF protein in SGNs, the release and translocation of AIF from the mitochondria resulted in high levels of nuclear and cytosolic AIF. As a result, although the contents of PARP-1 in the nuclei were similar among the three groups, the PAR polymer levels, a product of activated PARP-1, were increased in the AIF-knockdown group $(26,27)$. However, it remains unknown how AIF knockdown increase the levels of PAR, which conversely leads to AIF release.

In addition, although the expression levels of AIF in cochlea spiral tissue were found to be decreased over time during the stage of growth and development, they were 
shown to be increased, to a certain extent, in the elderly. The variation of AIF expression levels might be closely associated with the complicated regulatory roles of AIF expression at different physiological phases, be that its vital mitochondrial role in healthy cells or its lethal activity in aging-related cell dysfunctions (28).

As is well known, the X-linked AIFM1 gene encodes mitochondrial apoptosis inducing factor (AIF) is an FAD-containing and NADH oxidase and critically important for energy metabolism $(29,30)$. Many studies indicated that AIF may regulate mitochondrial function by participating in the assembly and/or stabilization of the respiratory complexes and contributed to the normal cellular functions. However, similar as other mitochondrial factors, the AIF can also be released from mitochondria during apoptosis, and then migrates to the nucleus, inducing chromatin condensation and DNA fragmentation by unknown molecular mechanism. As a result, the AIF, as seen in cytochrome c, may also has a bifunctional protein with dissociable apoptogenic and redox properties (31). Thus, we speculated that the AIF might play certain positive roles during the early growth and development stage. However, over time, the potential positive roles of AIF might gradually reduce, and the expression levels of AIF were decreased by unknown molecular mechanism. The decrease of AIF expression levels might lead to the AIF deficiency. The AIF further compromises oxidative phosphorylation and contributed to the dysfunction and apoptosis of targeted cells. But, as the rats get older, AIF might play much more important role in the apoptosis inducing, and the increase of some apoptotic factors might enhance the expression of AIF, yet with negative control results of AIF. We thought it will be important to investigate different potential roles of AIF during development and aging, in particular, to what extent and through which exact mechanisms contributes to regulation the expression levels of AIF as well as its exactly regulation roles. What's more, the new experiment methods, such as site-specific gene delivery system, new CRISPR/Cas 9 Technology or site injection of AIF-activating or AIF-inhibiting molecules could be used for AIF gene regulation (knockdown or overexpression) study as well as its in vivo potential regulation effects.

In conclusion, the present data revealed new regulatory effects of the AIF protein on SGNs dysfunctions in vitro. It was shown that AIF knockdown with siRNA resulted in high levels of oxidative stress status, impaired mitochondrial respiration activity and membrane potential in SGNs. Western blotting analysis further indicated that AIF knockdown could decrease the expression levels of anti-apoptotic and anti-oxidative executors, as well as mitochondrial respiratory chain Complex I proteins. This study provided a molecular basis for the development of new prevention and therapeutic strategies for ANSD.

\section{Acknowledgements}

Not applicable.

\section{Funding}

This study was financially supported by the National Natural Science Foundation of China (grant no. 81600795) awarded to LZ. The funders played no role in the study design, data collection and analysis, decision to publish, or preparation of the manuscript.

\section{Availability of data and materials}

The datasets generated and/or analyzed during the current study are not publicly available due to the institutional rules of the PLA General Hospital but are available from the corresponding author on reasonable request.

\section{Authors' contributions}

$\mathrm{LZ}$ and $\mathrm{JZ}$ performed the experiments and drafted the manuscript. WW designed the experiments. JW and DH conducted statistical analysis of the data. ML conceived the study, and participated in its design and coordination.

\section{Ethics approval and consent to participate}

All animal procedures performed on SPF SD rats were approved by the local Institutional Animal Care and Use Committee of the Chinese PLA General Hospital.

\section{Patient consent for publication}

Not applicable.

\section{Competing interests}

The authors declare that they have no competing interests.

\section{References}

1. Kaga K: Auditory nerve disease and auditory neuropathy spectrum disorders. Auris Nasus Larynx 43: 10-20, 2016.

2. Manchaiah VK, Zhao F, Danesh AA and Duprey R: The genetic basis of auditory neuropathy spectrum disorder (ANSD). Int J Pediatr Otorhinolaryngol 75: 151-158, 2011.

3. Gökdoğan Ç, Altınyay Ş, Gündüz B, Kemaloğlu YK, Bayazıt Y and Uygur K: Management of children with auditory neuropathy spectrum disorder (ANSD). Braz J Otorhinolaryngol 82: 493-499, 2016.

4. Moser T and Starr A: Auditory neuropathy-neural and synaptic mechanisms. Nat Rev Neurol 12: 135-149, 2016.

5. Pan H, Song Q, Huang Y, Wang J, Chai R, Yin S and Wang J: Auditory neuropathy after damage to cochlear spiral ganglion neurons in mice resulting from conditional expression of diphtheria toxin receptors. Sci Rep 7: 6409, 2017.

6. Yuste VJ,LorenzoHK and Susin S A: AIFM1 (Apoptosis-Inducing Factor, Mitochondrion-Associated, 1). Atlas of Genetics and Cytogenetics in Oncology and Haematology, pp190-194, 2008.

7. Bano D and Prehn JHM: Apoptosis-inducing factor (AIF) in physiology and disease: The tale of a repented natural born killer. EBioMedicine 30: 29-37, 2018.

8. Joza N, Oudit GY,Brown D, Bénit P, KassiriZ, Vahsen N, Benoit L, Patel MM, Nowikovsky K, Vassault A, et al: Muscle-specific loss of apoptosis-inducing factor leads to mitochondrial dysfunction, skeletal muscle atrophy, and dilated cardiomyopathy. Mol Cell Biol 25: 10261-10272, 2005.

9. Wang B, Li X, Wang J, Liu L, Xie Y, Huang S, Pakhrin PS, Jin Q, Zhu C, Tang B, et al: A Novel AIFM1 Mutation in a Chinese Family with X-linked Charcot-Marie-Tooth disease type 4. Neuromuscul Disord 28: 652-659, 2018.

10. Zong L, Guan J, Ealy M, Zhang Q, Wang D, Wang H, Zhao Y, Shen Z, Campbell CA, Wang F, et al: Mutations in apoptosis-inducing factor cause X-linked recessive auditory neuropathy spectrum disorder. J Med Genet 52: 523-531, 2015. 
11. Brors D, Bodmer D, Pak K, Aletsee C, Schäfers M, Dazert S and Ryan AF: EphA4 provides repulsive signals to developing cochlear ganglion neurites mediated through ephrin-B2 and -B3. J Comp Neurol 462: 90-100, 2010.

12. Aletsee C, Beros A, Mullen L, Palacios S, Pak K, Dazert S and Ryan AF: Ras/MEK but not p38 signaling mediates NT-3-induced neurite extension from spiral ganglion neurons. J Assoc Res Otolaryngol 2: 377-387, 2001.

13. Szabó Z, Harasztosi C, Szûcs G, Sziklai I and Rusznák Z: A detailed procedure and dissection guide for the isolation of spiral ganglion cells of the guinea pig for electrophysiological experiments. Brain Res Brain Res Protoc 10: 139-147, 2003.

14. Liu W, Fan Z, Han Y, Zhang D, Li J and Wang H: Intranuclear localization of apoptosis-inducing factor and endonuclease $G$ involves in peroxynitrite-induced apoptosis of spiral ganglion neurons. Neurol Res 34: 915-922, 2012.

15. ApostolovaN,Cervera AM,Victor VM,Cadenas S, Sanjuan-Pla A, Alvarez-Barrientos A, Esplugues JV and McCreath KJ: Loss of apoptosis-inducing factor leads to an increase in reactive oxygen species, and an impairment of respiration that can be reversed by antioxidants. Cell Death Differ 13: 354-357, 2006.

16. Jin Y, Kondo K, Ushio M, Kaga K, Ryan AF and Yamasoba T: Developmental changes in the responsiveness of rat spiral ganglion neurons to neurotrophic factors in dissociated culture: Differential responses for survival, neuritogenesis and neuronal morphology. Cell Tissue Res 351: 15-27, 2013.

17. Mendoza EE, Pocceschi MG, Kong X, Leeper DB, Caro J, Limesand $\mathrm{KH}$ and Burd R: Control of glycolytic flux by AMP-activated protein kinase in tumor cells adapted to low pH 1. Transl Oncol 5: 208-216, 2012.

18. Teng YD, Choi H, Onario RC, Zhu S, Desilets FC, Lan S, Woodard EJ, Snyder EY, Eichler ME and Friedlander RM: Minocycline inhibits contusion-triggered mitochondrial cytochrome c release and mitigates functional deficits after spinal cord injury. Proc Natl Acad Sci USA 101: 3071-3076, 2004.

19. Choi GE, Oh JY, Lee HJ, Chae CW, Kim JS, Jung YH and Han HJ: Glucocorticoid-mediated ER-mitochondria contacts reduce AMPA receptor and mitochondria trafficking into cell terminus via microtubule destabilization. Cell Death Dis 9: 1137, 2018.

20. Liu H, Zhang H, Wu X, Ma D, Wu J, Wang L, Jiang Y, Fei Y, Zhu C, Tan R, et al: Nuclear cGAS suppresses DNA repair and promotes tumorigenesis. Nature 563: 131-136, 2018.
21. Whitlon DS, Grover M, Tristano J, Williams T and Coulson MT: Culture conditions determine the prevalence of bipolar and monopolar neurons in cultures of dissociated spiral ganglion. Neuroscience 146: 833-840, 2007.

22. Murphy MP: How mitochondria produce reactive oxygen species. Biochem J 417: 1-13, 2009.

23. Otera H, Ohsakaya S, Nagaura Z, Ishihara N and Mihara K: Export of mitochondrial AIF in response to proapoptotic stimuli depends on processing at the intermembrane space. EMBO J 24: 1375-1386, 2014.

24. Vandenabeele P, Galluzzi L, Vanden Berghe T and Kroemer G: Molecular mechanisms of necroptosis: An ordered cellular explosion. Nat Rev Mol Cell Biol 11: 700-714, 2010.

25. Vahsen N, Candé C, Brière JJ, Bénit P, Joza N, Larochette N, Mastroberardino PG, Pequignot MO, Casares N, Lazar V, et al: AIF deficiency compromises oxidative phosphorylation. EMBO J 23: 4679-4689, 2014

26. Andrabi SA, Kim NS, Yu SW, Wang H, Koh DW, Sasaki M, Klaus JA, Otsuka T,Zhang Z, Koehler RC, et al: Poly(ADP-ribose) (PAR) polymer is a death signal. Proc Natl Acad Sci USA 103: 18308-18313, 2006.

27. Yu SW, Andrabi SA, Wang H, Kim NS, Poirier GG, Dawson TM and Dawson VL: Apoptosis-inducing factor mediates poly(ADP-ribose) (PAR) polymer-induced cell death. Proc Natl Acad Sci USA 103: 18314-18319, 2006.

28. Hangen E, Blomgren K, Bénit P, Kroemer G and Modjtahedi N: Life with or without AIF. Trends Biochem Sci 35: 278-287, 2010.

29. Susin SA, Lorenzo HK, Zamzami N, Marzo I, Snow BE, Brothers GM, Mangion J, Jacotot E, Costantini P,Loeffler M, et al: Molecular characterization of mitochondrial apoptosis-inducing factor. Nature 397: 441-446, 1999.

30. Miramar MD, Costantini P, Ravagnan L, Saraiva LM, Haouzi D, Brothers G, Penninger JM, Peleato ML, Kroemer G and Susin SA: NADH oxidase activity of mitochondrial apoptosis-inducing factor. J Biol Chem 276: 16391-16398, 2001.

31. Maté MJ, Ortiz-Lombardía M, Boitel B, Haouz A, Tello D, Susin SA, Penninger J, Kroemer G and Alzari PM: The crystal structure of the mouse apoptosis-inducing factor AIF. Nat Struct Biol 9: 442-446, 2002.

This work is licensed under a Creative Commons Attribution-NonCommercial-NoDerivatives 4.0 International (CC BY-NC-ND 4.0) License. 